\title{
Pupil and iris detection algorithm for near-infrared capture devices
}

\author{
Adam Szczepański ${ }^{1}$, Krzysztof Misztal $^{1}$ and Khalid Saeed ${ }^{1,2}$ \\ 1 AGH University of Science and Technology \\ Faculty of Physics and Applied Computer Science \\ al. A. Mickiewicza 30, PL-30059 Kraków, Poland \\ 2 Faculty of Computer Science \\ Bialystok University of Technology, \\ Wiejska 45A, PL-15351 Bialystok, Poland
}

\{Adam.Szczepanski,Krzysztof.Misztal,Khalid.Saeed\}@fis.agh.edu.pl

\begin{abstract}
In this paper a simple and robust solution for the pupil and iris detection is presented. The procedure is based on simple operations, such as erosion, dilation, binarization, flood filling and Sobel filter and, with proper implementation, is effective. The novelty of the approach is the use of distances of black points from nearest white points to estimate and then adjust the position of the center and the radius of the pupil which is also used for iris detection. The obtained results are promising, the pupil is extracted properly and all the information necessary for human identification and verification can be extracted from the found parts of the iris. The paper, being both review and research, contains also a state of the art in the described topic.
\end{abstract}

Keywords: iris detection, pupil detection, gradient analysis, linear analysis

\section{Introduction}

With the open borders and the ease of international travel human identification and verification is nowadays more important than ever before. On one hand it is essential for public safety, although on the other hand, people tend to dislike invasive methods of control. There are many biometric solutions that try to address this issue and all of them have their advantages and disadvantages. For example fingerprint images, which are commonly used as a biometric feature in modern identification documents, are easily obtainable although they are very vulnerable to identity theft. Also most of fingerprint scanners have to be touched which brings hygiene problems. For security reasons the iris image is the best option, although its acquisition is much more comfortable.

The iris, on the other hand, combines both high level of resistance for identity theft. It is hard to damage as the iris is an internal organ and is well protected by a highly transparent and sensitive membrane. It is different even between identical twins, does not change during the whole life and decays only a few minutes after death. Also the physiological reaction of the iris towards the light 
and the natural movement of the pupil makes it impossible to replace the iris tissue with a photograph. The iris is also easy to acquire - the person needs just to look in the direction of the camera. This factors make the iris a reasonable choice of biometric feature both for identification and verification usages.

In this work the system of pupil and iris acquisition for such purposes is described. As this paper is both research and review, the first section of the paper contains a state of the art in described matter. Then the proposed solution is described along with the results of the tests of the algorithm. The last section contains conclusions and the description of future work.

\section{State of the art}

While iris pattern is one of the most reliable biometric form of human identification, many methods have been developed for automatic iris recognition [1]. It is enough to mention following algorithms:

- the Gabor wavelet approach by Daugman [2],

- the Laplacian parameter approach by Wildes [3],

- zero-crossings of the wavelet transform at various resolution levels by Boles et al. [4],

- the Independent Component Analysis approach by Huang et al. [5],

- the texture analysis using multi-channel Gabor filtering and wavelet transform by Zhu et al. [6], and many others.

Iris segmentation and localization are one of the crucial steps in every of then. An iris-recognition algorithm first has to identify the approximately concentric circular outer boundaries of the iris and the pupil in a photo of an eye. One has often to deal with additional problems caused by the eyelids which can reduce the surface of the iris. In that case the mask template is also formed, which help to take into account bad pixel in iris feature generating and iris comparison.

Lets now look close on two of them [7].

John Daugman's algorithm uses the integro-differential operator for determining the localization of the circular iris and pupil in addition to the areas of the upper and lower eyelids. The operator is defined as follows:

$$
\max _{r, x_{0}, y_{0}}\left|G_{\sigma}(r) * \frac{\partial}{\partial r} \oint_{r, x_{0}, y_{0}} \frac{I(x, y)}{2 \pi r} d s\right|
$$

where:

- I(x,y) - image (containing the eye) pixel color,

- $G_{\sigma}(r)$ - the Gaussian smoothing function with scale $(\sigma)$ is given by the following formula

$$
G_{\sigma}(r)=\frac{1}{\sqrt{2 \pi} \sigma} e^{-\left(r-r_{0}\right)^{2} / 2 \sigma^{2}}
$$

$-r$ - searching radius,

$-s$ - contour given by circle with $r, x_{0}, y_{0}$ parameters. 
The smoothed image is scanned for a circle that has the maximum gradient change that indicates an edge. To obtain a precise localization the operator, is applied iteratively. During this operation the smoothing function scale is changed to increase the accuracy. This not ends the algorithm, because we want to localize eyelids, which is done in similar way. The contours is changed from a circular into an arc.

The main drawback of this method lies on noise sensitivity. Thus it can failed on non-ideal environmental condition [8].

The second approach given by Wildes et al. algorithm perform localization and segmentation in two steps. Firstly the image is converted into edge-map we extract information about the edge points in the image. One of the possible method to do this is thresholding the magnitude of the image intensity gradient:

$$
\left|\nabla G_{\sigma}(x, y) * I(x, y)\right|,
$$

where $\nabla=(\partial / \partial x, \partial / \partial y)$ and the $G_{\sigma}$ is two-dimensional Gaussian with center $\left(x_{0}, y_{0}\right)$ and standard derivation $\sigma$ that smooth the image. We can alternatively use various other method in this step, ex. Sobel or Canny [9]. Second step allows us to select circles describing ex. pupil using voting procedure on parametric definitions of the iris boundary contours. which can be realized via Hough transforms [10].

By the knowledge that the eyelids are usually horizontally aligned Wildes [11] performing the preceding edge detection step with bias the derivatives in the horizontal direction for detecting the eyelids, and in the vertical direction for detecting the outer circular boundary of the iris. Consequently the eyelid edge map will corrupt the circular iris boundary edge map if all gradient data would be used. Taking only the vertical gradients for locating the iris boundary will reduce influence of the eyelids when performing circular Hough transform, and not all of the edge pixels defining the circle are required for successful localization.

However also in this case methods have same drawback. Since the Wildes' approach is based on intensive image processing, effects leading to alterations in the grey value distribution can influence the result negatively. Moreover Hough transform require high computation time (the complexity of fastest implementation is $O\left(N^{3}\right)$ in the number of considered (edge) points on the image [12]).

\section{Proposed algorithm}

The algorithm was developed for the purpose of analysis of images captured by dedicated devices. The procedure is presented in Fig. 1.

The solution is split into four parts:

- Initial preprocessing

- Pupil acquisition

- Preprocessing for iris acquisition

- Iris acquisition

The second and third parts can be conducted simultaneously if multi-core solution is available. 


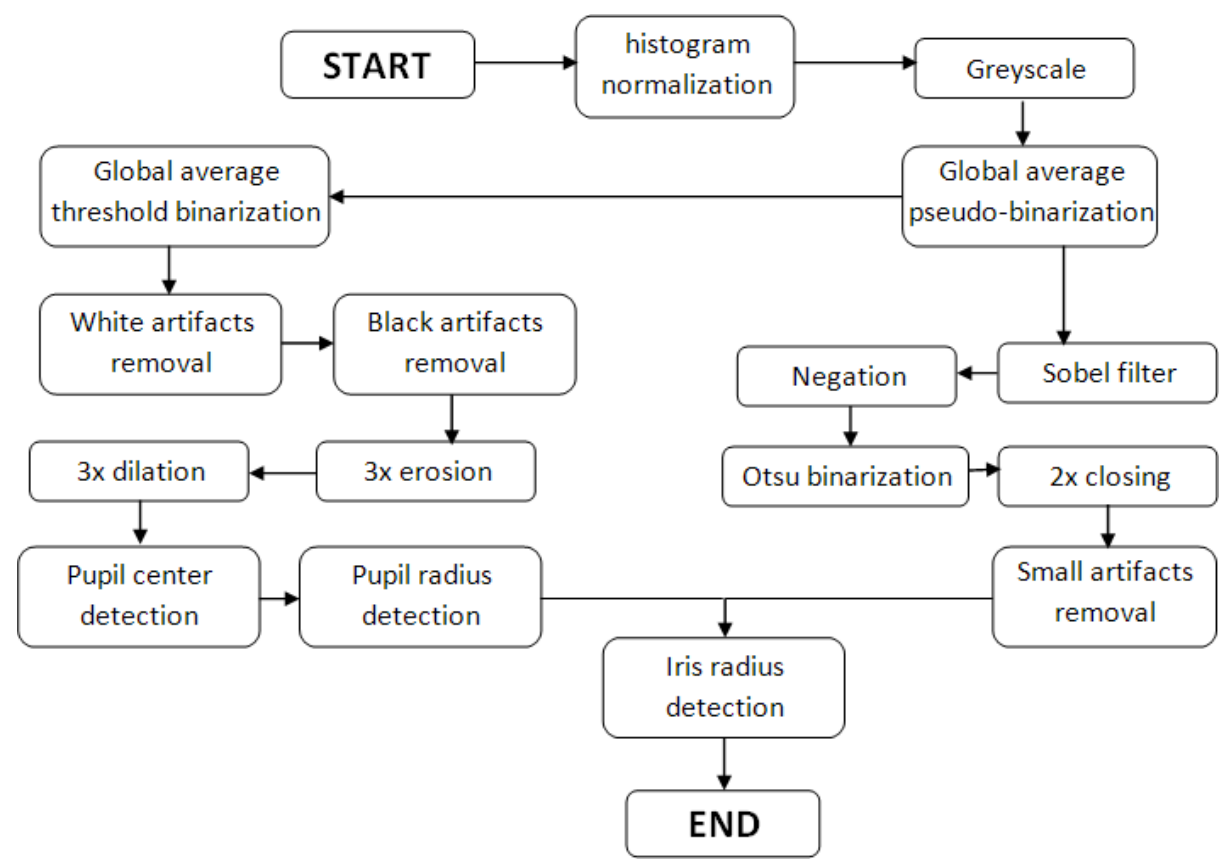

Fig. 1: Flowchart of the algorithm.

\subsection{Initial preprocessing}

Initial preprocessing consists of three basic steps which are conducted to normalize the data and reduce unnecessary information on the images. The first step is the normalization of histogram where the new value of pixel $\mathrm{P}(\mathrm{x}, \mathrm{y})$ for each separate color channel of RGB palette is calculated using:

$$
P(x, y)=255 \cdot \frac{P(x, y)-\min P}{\max P-\min P}
$$

Where $\min P$ and $\max P$ are the maximum and minimum values of each channel. The second step is conversion of the image into gray scale by averaging the RGB values. The third step, which is called global average pseudo-binarization in Fig. 1 is conducted similarly to regular binarization with global average, however only the pixels which are above average are converted into white pixels, the pixels below or equal average are left unchanged. This step, as presented in Fig. 2 , removes lighter fragments of the pictures, such as eyelids or parts of eyeballs, from further analysis. This step also improves the results of Sobel filter used in iris acquisition and alters the threshold of global binarization during pupil acquisition where only non-white pixels are analyzed. 

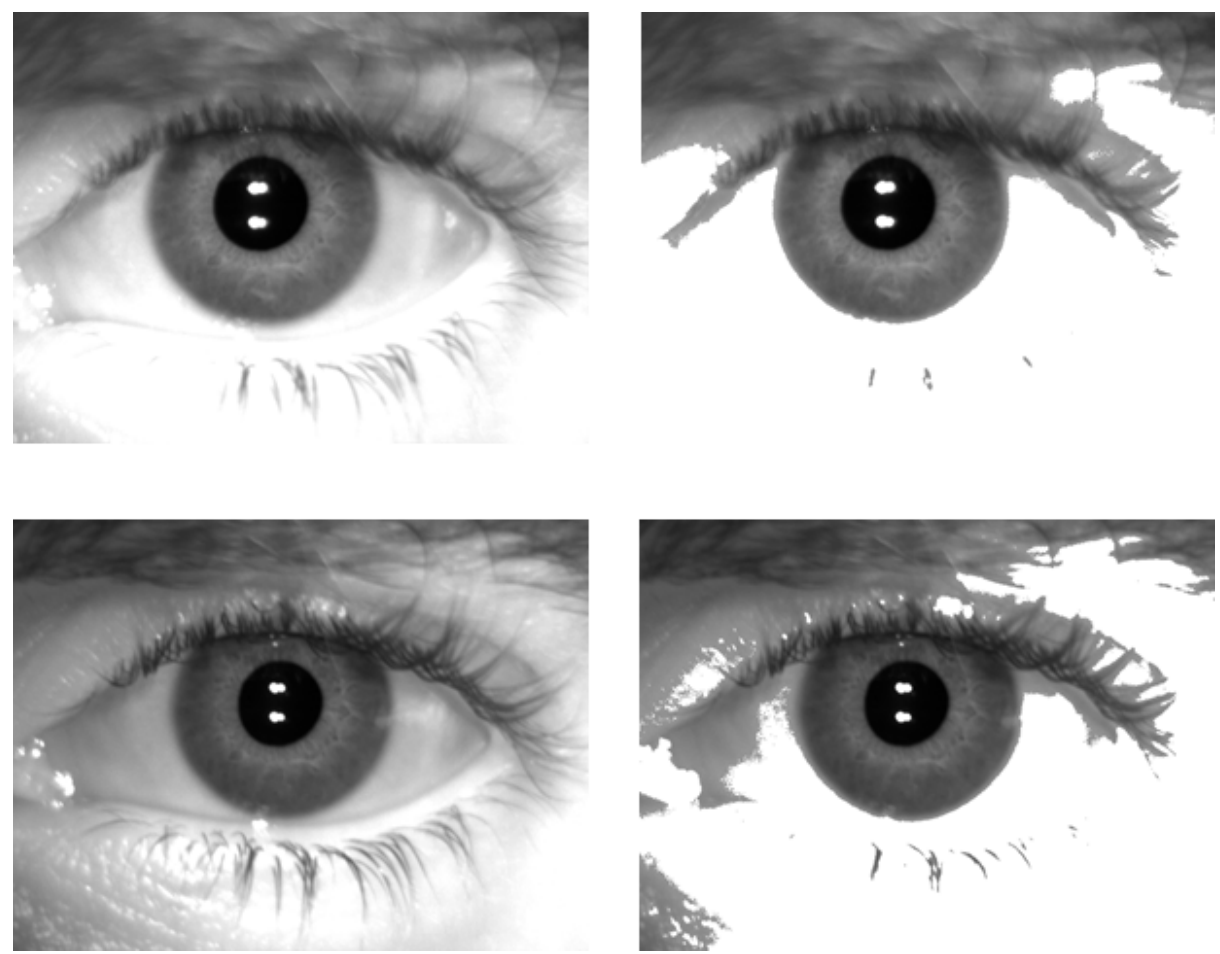

Fig. 2: Exemplary results of global average pseudo-binarization, on the left are original images and on the right are images after first step of proposed algorithm.

\subsection{Pupil acquisition}

This part of algorithm starts with binarization using $20 \%$ of global average as a threshold. The average is calculated using only non-white pixels of the image. Each pixel above this value is converted into black pixel and the rest are converted into white pixels. This operation, as presented in Fig. 3, leaves relatively good pupil shape with some minor artifacts. The white gaps inside the black shapes are filled using flooding algorithm and then all but the largest black shapes are converted into white ones using flooding algorithm again. The results of this operations are also presented in Fig. 3. Next the image is eroded three times and dilated three times to prepare it for pupil acquisition.

The first step of the acquisition is the calculation of the distances of every black pixel from the nearest white pixel. Then the coordinates of the initial center of the pupil are calculated by averaging the coordinates of the points with the longest distances. Having the initial central point, the radius of the pupil is acquired as described with pseudo-code below:

1. Calculate the rectangular borders of the shape

2. Calculate distances from the center point to the furthest left, right, top and bottom black pixel horizontally and vertically 

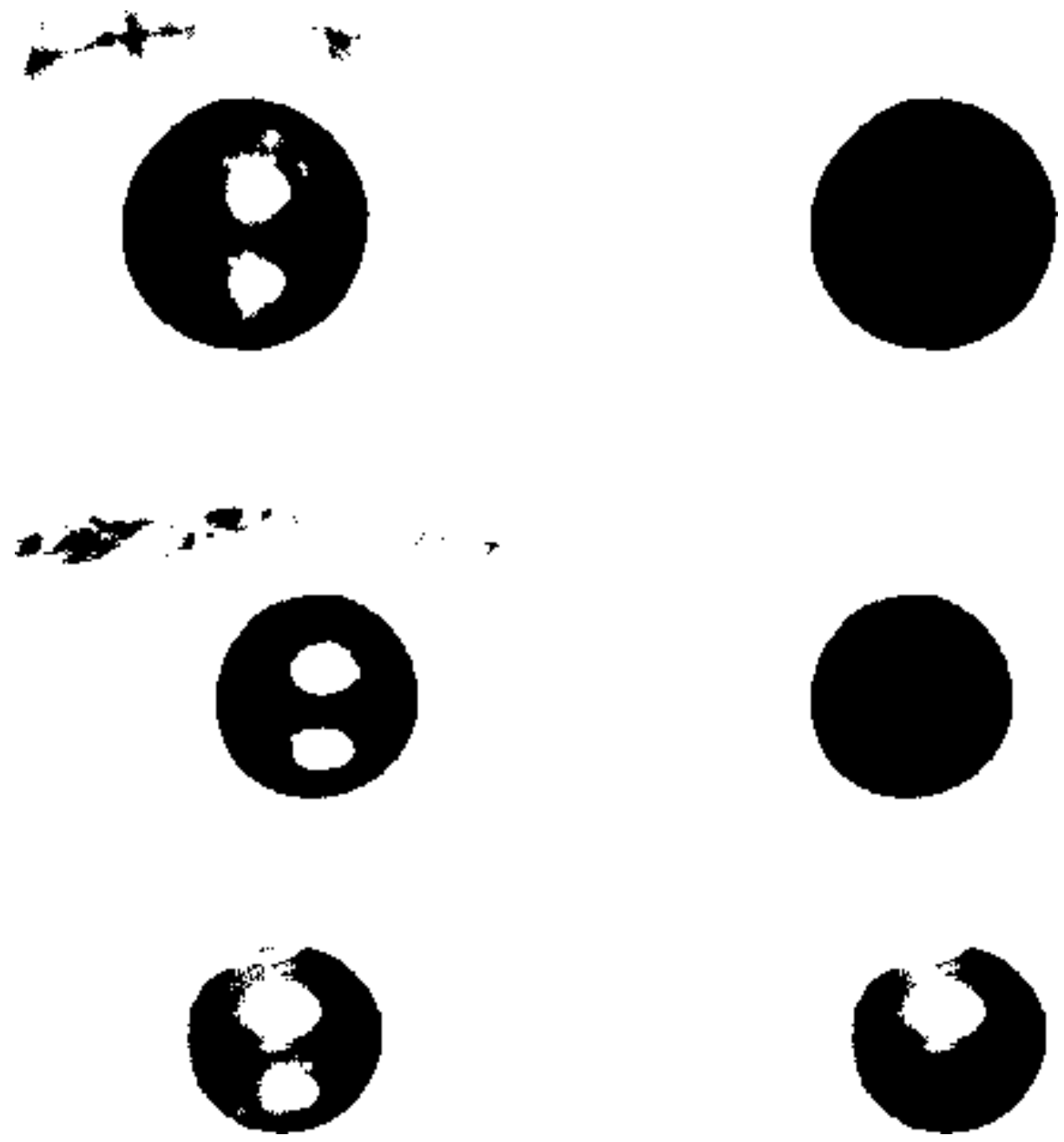

Fig. 3: Exemplary results of thresholding (left) and flooding algorithm (right).

3. Check which span is wider - between left and right pixel or between top and bottom

4. Calculate the radius as a half of the wider span

5. If it is not the first iteration of the loop and the radius is shorter than the radius calculated in the previous step - end the procedure

6. Calculate the difference between one of the distances of wider span and the radius and adjust the center point by this difference in the wider span direction (vertical or horizontal)

7. Calculate the distances of the moved center point from the borders of the shape in the shorter span direction 
8. Choose the shorter distance. If it is left or bottom, the new value of center point in the shorter direction is the border point value in that direction + radius, else it is the border point value in that direction - radius

After this procedure the center and the radius of the pupil are acquired and can be used in the Iris acquisition step.

\subsection{Preprocessing for iris acquisition}

Separately from step two, the image is also prepared for iris acquisition. This step can be implemented simultaneously with previous step on multi-core systems. It begins with Sobel filtering and the negation of the filtering result. Then all the black pixels in the image are converted into white ones. This step eliminates the strong borders which separate the white and non-white regions of the image after Sobel filtering. Then the image is binariezed using Otsu algorithm. After binarization the closing operation is performed twice and the removal of artifacts is conducted using flooding algorithm. With this step all black shapes that consist the number of pixels lesser than $0.2 \%$ of total pixel count of the image are whitened.

The image is now prepared for iris acquisition using the pupil obtained in step 2.

\subsection{Iris acquisition}

To acquire the iris, the eight lines are drawn from the center of the pupil. The lines start in the distance of $1.5 \times$ pupil radius from the center and are drawn horizontally to the left and right, vertically to the top and bottom and in the 30, 150, 210 and 330 degrees from the horizontal line. Each line ends when it meets the first black pixel or reaches the border of the image, as presented in Fig. 4. The distances between first found points and the center point are calculated and sorted. The median of these distances is used as the radius of the iris.

\section{Results}

The tests were conducted on 47 sample images from VeriEye SDK acquired using Retica Mobile-Eyes Ruggedized Dual Iris Capture Device. The images are compatible with ANSI INCITS 379-2004 and ISO/IEC 19794-6 standards. The database consists of pictures of both left and right eyes of 10 people. The number of samples of each person and each eye varies. The database was chosen due to the variety of iris positions and lighting conditions on the samples.

All the pupils were extracted correctly. In some cases the region of the found iris is larger than actual one although all the parts of the iris needed for human recognition and verification in each case were acquired. The exemplary results are presented in Fig. 5. In overall, the success rate of this initial tests was 100\% although the authors are aware that more thorough tests are still required. 

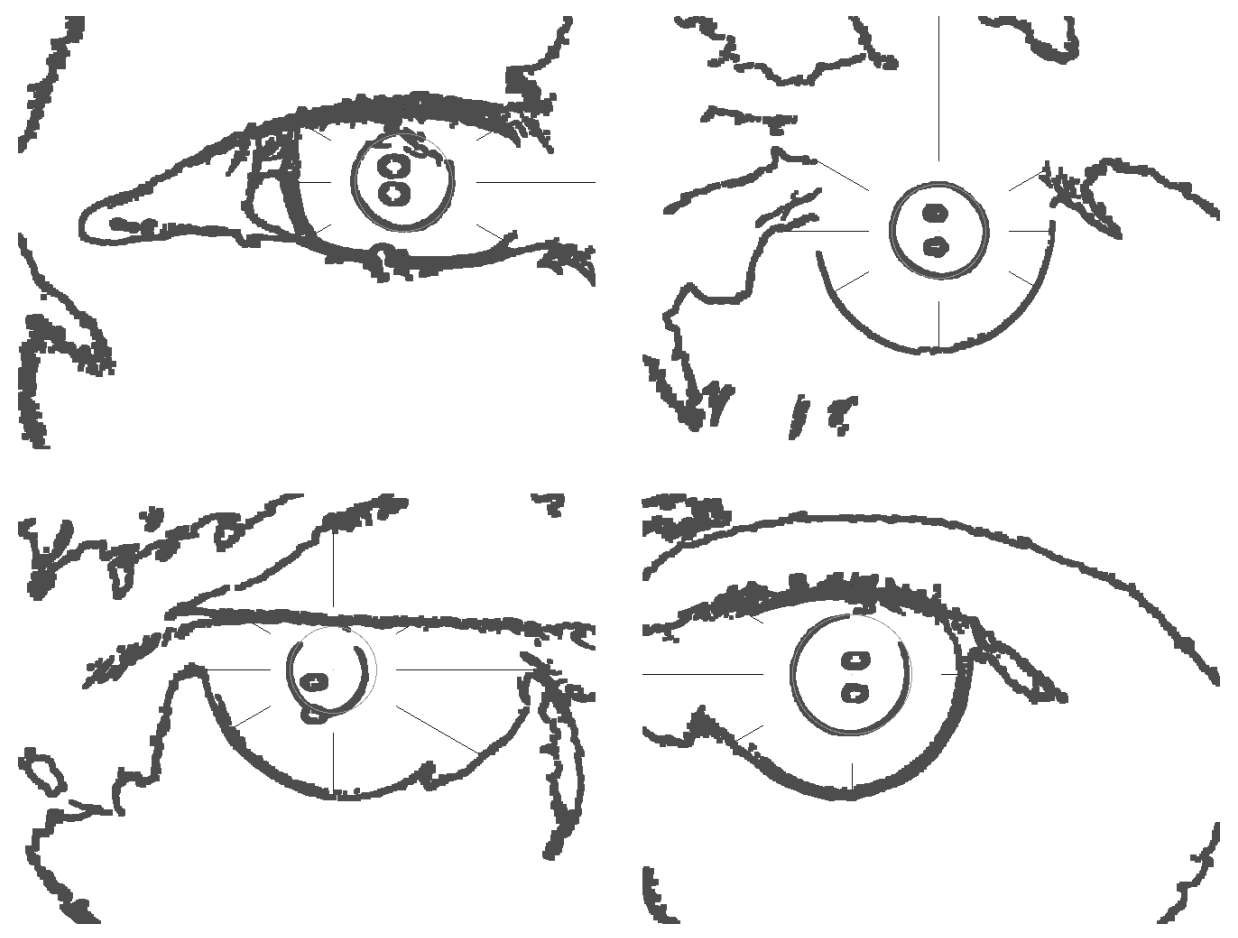

Fig. 4: Exemplary results of iris radius detection.

\section{Conclusions and future work}

As mentioned above, the results of the experiments are promising. The algorithm sometimes acquires redundant information, although the extra acquired iris neighborhood can be easily eliminated during a transformation of the iris into a rectangle. This neighborhood will transform into much lighter then the iris part of the strip and on this stage simple thresholding should be enough to address the issue.

Noticeable is that the algorithm handled correctly also the images which were overexposed.

The main disadvantage of the solution is the extensive use of dilation and erosion algorithms which, when poorly implemented, may be time-consuming. The authors recommend the use of calculation of distances to nearest white/black pixel in this process to reduce the computation time of the algorithm.

In their future work the authors will concentrate on the second part of the planned solution - the acquisition of characteristic features of the iris. The aforementioned transformation of the iris into a rectangle will be used for this purpose and any other necessary preprocessing will be conducted in this part of the system. Then the authors will work on the development of human identification and verification procedures to propose a complete solution. 

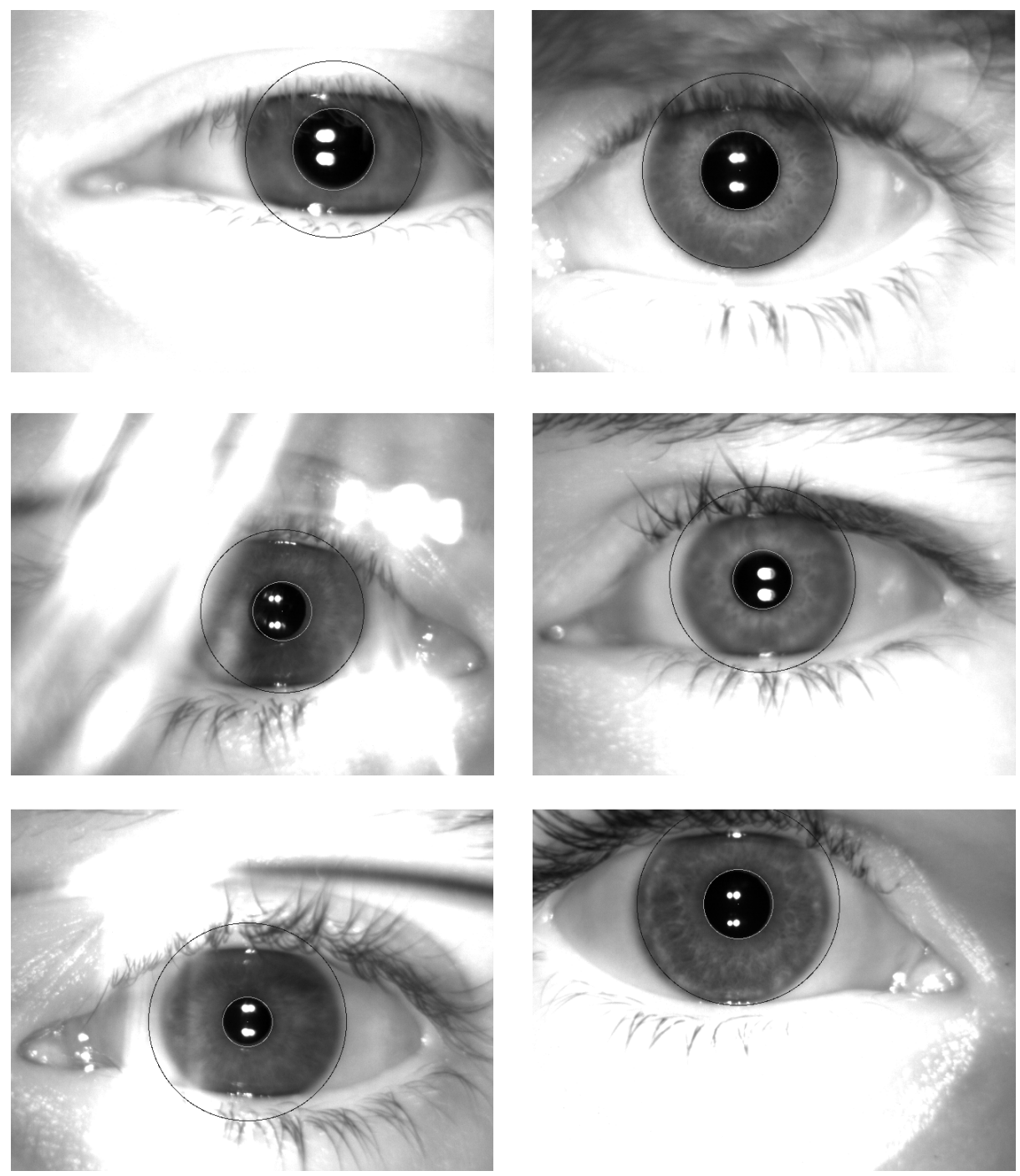

Fig. 5: The exemplary results.

\section{Acknowledgement}

The research of Krzysztof Misztal is supported by the National Centre of Science (Poland) [grant no. 2012/07/N/ST6/02192].

\section{References}

1. Masek, L., et al.: Recognition of human iris patterns for biometric identification. M. Thesis, The University of Western Australia 3 (2003) 
2. Daugman, J.G.: High confidence visual recognition of persons by a test of statistical independence. Pattern Analysis and Machine Intelligence, IEEE Transactions on 15 (1993) 1148-1161

3. Wildes, R.P.: Iris recognition: an emerging biometric technology. Proceedings of the IEEE 85 (1997) 1348-1363

4. Boles, W., Boashash, B.: A human identification technique using images of the iris and wavelet transform. Signal Processing, IEEE Transactions on 46 (1998) $1185-1188$

5. Huang, Y.P., Luo, S.W., Chen, E.Y.: An efficient iris recognition system. In: Machine Learning and Cybernetics, 2002. Proceedings. 2002 International Conference on. Volume 1., IEEE (2002) 450-454

6. Zhu, Y., Tan, T., Wang, Y.: Biometric personal identification based on iris patterns. In: Pattern Recognition, 2000. Proceedings. 15th International Conference on. Volume 2., IEEE (2000) 801-804

7. Misztal, K., Saeed, E., Tabor, J., Saeed, K. In: Iris Pattern Recognition with a New Mathematical Model to Its Rotation Detection. Springer New York (2012) 43-65

8. Jillela, R., Ross, A.A.: Methods for iris segmentation. In: Handbook of Iris Recognition. Springer (2013) 239-279

9. Gonzalez, R.C., Woods, R.E.: Digital image processing (2002)

10. Illingworth, J., Kittler, J.: A survey of the hough transform. Computer vision, graphics, and image processing 44 (1988) 87-116

11. Wildes, R.P., Asmuth, J.C., Green, G.L., Hsu, S.C., Kolczynski, R.J., Matey, J., McBride, S.E.: A system for automated iris recognition. In: Applications of Computer Vision, 1994., Proceedings of the Second IEEE Workshop on, IEEE (1994) $121-128$

12. Xie, Y., Ji, Q.: A new efficient ellipse detection method. In: Pattern Recognition, 2002. Proceedings. 16th International Conference on. Volume 2., IEEE (2002) 957960 